\title{
A Sensitivity Function Approach to the Design of Rudder Roll Stabilization Controller
}

\author{
Ching-Yaw Tzeng \\ Professor, Institute of Maritime Technology, National Taiwan Ocean University, Keelung, Taiwan, R.O.C. \\ Chung-Yi Wu \\ Graduate Students, Institute of Maritime Technology, National Taiwan Ocean University, Keelung, Taiwan, R.O.C. \\ Yu-Lung Chu \\ Graduate Students, Institute of Maritime Technology, National Taiwan Ocean University, Keelung, Taiwan, R.O.C.
}

Follow this and additional works at: https://jmstt.ntou.edu.tw/journal

Part of the Engineering Commons

\section{Recommended Citation}

Tzeng, Ching-Yaw; Wu, Chung-Yi; and Chu, Yu-Lung (2001) "A Sensitivity Function Approach to the Design of Rudder Roll Stabilization Controller," Journal of Marine Science and Technology. Vol. 9: Iss. 2, Article 5.

DOI: 10.51400/2709-6998.2440

Available at: https://jmstt.ntou.edu.tw/journal/vol9/iss2/5

This Research Article is brought to you for free and open access by Journal of Marine Science and Technology. It has been accepted for inclusion in Journal of Marine Science and Technology by an authorized editor of Journal of Marine Science and Technology. 


\title{
A SENSITIVITY FUNCTION APPROACH TO THE DESIGN OF RUDDER ROLL STABILIZATION CONTROLLER
}

\author{
Ching-Yaw Tzeng*, Chung-Yi Wu** and Yu-Lung Chu**
}

\begin{abstract}
Keywords: rudder roll stabilization (RRS), internal model control, saturation, slew rate limitation, output sensitivity function, reference conditioning technique.
\end{abstract}

\begin{abstract}
This paper is concerned with the design of a rudder roll stabilization (RRS) system by the internal model control (IMC) method. The proposed method directly shapes the output sensitivity function which relates the wave disturbance to the ship roll motion, to achieve good disturbance rejection. Under the IMC structure, the sensitivity functions depend linearly on a design transfer function and this makes it easy to satisfy the internal stability condition. A reference conditioning technique is employed in the design that takes the limited actuator authority into the controller design consideration to avoid unknowingly pushing the actuator beyond its saturation and slew rate limitation boundaries. Numerical results indicate that very good roll reduction is achieved for sinusoidal disturbance and reasonable roll reduction is obtained for a narrow band type of disturbance generated by passing a white noise sequence through a second order shaping filter.
\end{abstract}

\section{INTRODUCTION}

Ship roll stabilization has been an area of active research since 1970s. This is because the roll motion greatly effects the comfort of the crew and the operation of onboard equipment. The antirolling water tanks and the fin stabilizers are the best known roll stabilizing devices in use [1]. However these approaches typically involve complicated mechanisms and also require extra space. Consequently, alternative approach based on utilization of existing equipment such as the rudder on board ship seems to be quite attractive [2].

The main purpose of the rudder is to control the yaw motion; that is, the heading of the ship in coursekeeping or course-changing maneuvers [3]. However,

Paper Received June 30, 2001. Author for Correspondence: C. Y. Tzeng. *Professor, Institute of Maritime Technology, National Taiwan Ocean University, Keelung, Taiwan, R.O.C.

**Graduate Students, Institute of Maritime Technology, National Taiwan Ocean University, Keelung, Taiwan, R.O.C. it is observed that there is an inward roll induced by the rudder immediately after initialization of the turning maneuver. The rudder roll stabilization (RRS) concept is based on utilization of the rudder induced inward roll, which is known as the undershoot behavior that characterizes the so-called nonminimum phase system with the existence of right-half-plane zeros in the corresponding transfer function [4].

To avoid interference with the heading control of the ship, and to achieve effective roll reduction, the bandwidth of the rudder roll control system must be higher than that of the rudder yaw control system, which implies that the rudder slew rate limit should be high enough to achieve satisfactory roll reduction performance [5]. Thus, the RRS is aiming at reducing the high frequency wave induced roll motion rather than the low frequency steady state heeling. This is quite different from the purpose of a heading control autopilot, where compensating for the low frequency yaw motion is of main concern [3].

Many design methods have been applied to the design of the RRS system. For instance, the LQG method [6], the $H \infty$ control method [7], the adaptive LQ method [8] and the robust control design method [9] have been proposed. Commercial product that incorporating the RRS and fin roll stabilization in forming an integrated roll reduction system has been proposed [10]. RRS for naval vessels has been extensively studied and satisfactory roll reduction performance has been reported [11].

The main difficulty of the rudder roll stabilization system lies on the limited rudder angle and rudder rate that may cause performance degradation and even cause system instability, if they are not properly taken care of [3]. Automatic gain reduction has been proposed in dealing with the slew rate limitation problem [12]. An improved control method has been proposed that combines a predictive control and a classical feedback control in forming a hybrid RRS system. Consistent performance under various sea states are reported with relatively low demand on the required rudder rate [13].

To maximize the roll stabilization performance, 
without causing instability to the system, the saturation (SAT) and slew rate limitation (SRL) have to be taken into design consideration. A reference conditioning technique will be employed that modifies the reference input such that the rudder command is always equal to the actual input into the plant [14]. This allows full utilization of the limited actuator power, without pushing beyond the SAT and SRL boundaries unknowingly.

Since the disturbance rejection performance of the RRS system depends on the sensitivity function that reveals the effect of the disturbance on the roll motion of the ship, it is reasonable to directly shape the output sensitivity function under the internal model control (IMC) framework. The IMC design method is characterized by a model-based approach that provides explicit relationship between the structure of the controller and that of the plant model. Moreover, the controller can be conveniently parameterized in terms of a speed of response parameter, which allows the user to tune the controller to meet specific control objective [15].

Numerical simulations with respect to a ship model reported in [5] will be conducted to evaluate the performance of the proposed design method.

\section{EQUATIONS OF MOTION AND DISTURBANCE MODELING}

\section{A. Equations of Motion}

It is well-known that to fully represent a rigid body motion in space requires a six-degree-of-freedom approach. However, for slow merchant ships like the large oil tankers, it is often assumed that the ship maneuvering motion can be regarded as a three-degreeof-freedom horizontal plane rigid body motion, which includes surge (translation along the $x$ axis), sway (translation along the $y$ axis) and yaw (rotation about the $z$ axis) (see Fig. 1). However, for high speed container ships or naval vessels like the frigates, the rudder induced roll motion (rotation about the $x$ axis) is not negligible. Hence, it is necessary to include the roll mode into the surge-sway-yaw-based maneuvering equations.

The equations of motion describing the dynamics of a ship are readily obtained from Newton's law in space-fixed coordinate $x_{0}-y_{0}$ (see Fig.1). However, to take advantage of the symmetry property of a ship, a ship-fixed coordinate system $x-y$ is preferred. With the origin for the axis system taken at the center of gravity of the ship, the steering equations of motion within ship-fixed coordinate system can be written as

$$
m(\dot{u}-v r)=X
$$

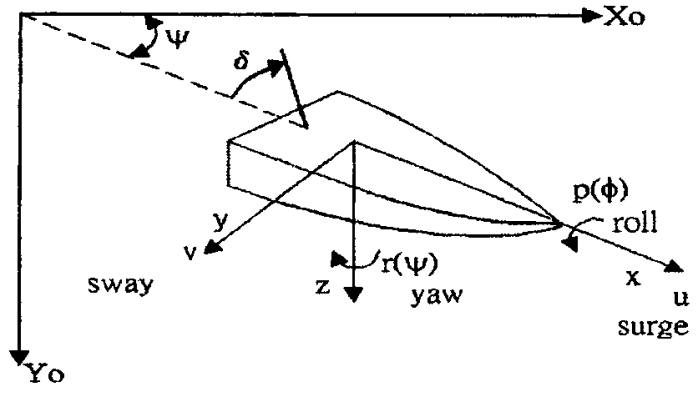

Fig. 1. Sway-yaw-roll motion coordinate system.

$$
\begin{aligned}
& m(\dot{v}+u r)=Y \\
& I_{z} \dot{r}=N \\
& I_{x} \dot{p}=K
\end{aligned}
$$

where

$m:=$ the mass of the ship ,

$u:=$ the surge speed; (speed in the $x$ direction),

$v:=$ the sway speed; (speed in the $y$ direction),

$p:=$ the roll rate; (angular rate about the $x$ axis),

$r:=$ the yaw rate; (angular rate about the $z$ axis),

$I_{z}:=$ the moment of inertia of the ship about the $z$ axis,

$X:=$ the force applied on the ship in the $x$ direction,

$Y:=$ the force applied on the ship in the $y$ direction,

$N:=$ the moment applied on the ship about the $z$ axis,

$K:=$ the moment applied on the ship about the $x$ axis.

The forces $X$, and $Y$, and moments $K$, and $N$ can be expressed as functions of the states $u, v, r, p$, their time derivatives $\dot{u}, \dot{v}, \dot{r}, \dot{p}$ and the rudder angle $\delta$. Hence,

$$
\begin{aligned}
& X=X(u, v, r, \dot{u}, \dot{v}, \dot{r}, \phi, p, \dot{p}, \delta) \\
& Y=Y(u, v, r, \dot{u}, \dot{v}, \dot{r}, \phi, p, \dot{p}, \delta) \\
& N=N(u, v, r, \dot{u}, \dot{v}, \dot{r}, \phi, p, \dot{p}, \delta) \\
& K=K(u, v, r, \dot{u}, \dot{v}, \dot{r}, \phi, p, \dot{p}, \delta)
\end{aligned}
$$

The state $\phi$ is the roll angle, and it is the integration of the roll rate $p$.

As the relationships between $X, Y, N$ and $K$ and the state variables are difficult to determine, Taylor series expansion has been suggested to expand the right hand sides of Eqs. (2) while keeping only the linear terms [1]. Substitution of the expansion terms into Eqs. (1) and note that upon linearization with respect to a straight line motion with a constant forward speed $u_{0}$, the surge equation is decoupled and the following linearized coupled sway-yaw-roll equations follow 


$$
\begin{aligned}
m\left(\dot{v}-u_{0} r\right) & =Y_{V} V+Y_{\dot{v}} \dot{V}+Y_{\varphi} \varphi+Y_{P} P+Y_{\dot{P}} \dot{P}+Y_{r} r \\
& +Y_{\dot{r}} \dot{r}+Y_{\delta} \delta \\
I_{x} \ddot{\varphi} & =K_{P} P+K_{\dot{P}} \dot{P}-m g \overline{G M} \phi+K_{V} V+K_{\dot{V}} \dot{V}+K_{r} r \\
& +K_{\dot{r}} \dot{r}+K_{\delta} \delta \\
I_{z} \ddot{\psi} & =N_{r} r+N_{\dot{r}} \dot{r}+N_{\phi} \phi+N_{P} P+N_{\dot{P}} \dot{P}+N_{V} V \\
& +N_{\dot{V}} \dot{V}+N_{\delta} \delta
\end{aligned}
$$

where $Y_{V}, Y_{\dot{v}}, \ldots .$. indicate the hydrodynamic coefficients; for instance, $Y_{V}$ indicates the derivative of the sway force $Y$ to the sway speed $V$ evaluated at the reference condition, and $\overline{G M}$ is the metacentric height, which indicates the restoring capability of a ship in roll motion.

Taking the Laplace transform of Eqs. (3a)-(3c), and after eliminating the sway speed $V$ and yaw rate $r$, the following transfer function relating the roll angle $\phi$ to the rudder angle $\delta$ can be obtained:

$$
\frac{\Phi}{\delta}=\frac{K_{1}\left(1+T_{3} s\right)\left(1+T_{4} s\right)}{\left(s^{2}+2 \zeta \omega_{n} s+\omega_{n}^{2}\right)\left(1+T_{1} s\right)\left(1+T_{2} s\right)}
$$

where the terms $\left(1+T_{1} s\right),\left(1+T_{2} s\right),\left(1+T_{3} s\right)$ and $(1+$ $T_{4} s$ ) are due to coupling effects from the sway and yaw motions.

In practice, it can be assumed that the sway speed $V$ is proportional to the yaw rate $\mathrm{r}$ during a turning maneuver [3]. This assumption seems to be well justified since it has been observed that during a turning maneuver, the sway speed $V$ and yaw rate r show very similar patterns, which is closely-related to the socalled cancellation effect [16].

Consequently, Eq. (4) is further reducible to the following form:

$$
\frac{\Phi}{\delta}=\frac{K_{0}\left(1+T_{6} s\right)}{\left(s^{2}+2 \zeta \omega_{n} s+\omega_{n}^{2}\right)\left(1+T_{5} s\right)}
$$

where the sway-yaw linear dependency relationship has been absorbed into the coefficient $K_{0}$.

System identification techniques have been applied to identify the roll-rudder transfer function based on sea trial data. Practical identification experiences indicate that direct identifying Eq. (4) would result in an ill-conditioning problem, which once again, can be attributed to the cancellation effect reported in [16]. Hence, it is often necessary to directly identify the simplified transfer function defined by Eq. (5). The following numerical values are obtained from identification results based on sea trial data of a warship [5]. We have

$$
\frac{\Phi}{\delta}=\frac{K_{0}(1-4.5 s)}{(1+8.2 s)\left(s^{2}+0.25 s+0.25\right)}
$$

where $K_{o}$ is a speed dependent gain coefficient, specifically, $K_{0}=0.140$ for a speed of $18 \mathrm{kt}$. Eq. (6) will be taken as the plant model $\widehat{G}$ to be used in the modelbased IMC design method described in latter section. It is seen that the zero corresponding to the numerator dynamics of Eq.(6) is a positive value; namely, on the right half plane and this corresponds to the so-called nonminimum phase system.

Fig. 2 gives the frequency response diagram of the rudder-roll transfer function defined by Eq. (6). A resonant peak at $\omega=0.5 \mathrm{rad} / \mathrm{sec}$ indicates the oscillatory characteristics of the rudder-roll system.

With the roll-rudder transfer function defined by Eq. (6), starboard turning maneuvers are conducted for $\delta=-20^{\circ}$ and $-30^{\circ}$. The numerical results are given in Fig. 3. It is seen that there is an initial undershoot, which corresponds to the rudder-induced inward roll and the larger is the rudder angle, the larger the undershoot is. Besides, the steady state outward roll angle increases linearly with respect to the applied rudder angle. This initial undershoot phenomenon characterizes the so-called nonminimum phase system, where a right half plane zero exists in the system transfer function.

\section{B. Hydrodynamic aspect of the rudder- induced roll}

Referring to Fig. 4 the rudder induced force $F_{R}$ appears immediately, once the rudder has reached the starboard position. The rudder force $F_{R}$ now generates a clockwise moment $M_{R}$ that rolls the ship inwards. It should be noted that the rudder induced force $F_{R}$ also results in a sideway kick phenomenon.

Since the size of the ship is significantly larger than that of the rudder, the hull induced force $F_{H}$ due to existence of drift angle built up much slowly. However once $F_{H}$ comes in, a counter clockwise moment $M_{H}$ is generated that outweighs the rudder-induced moment $M_{R}$ and an outward roll is generated. When the ship

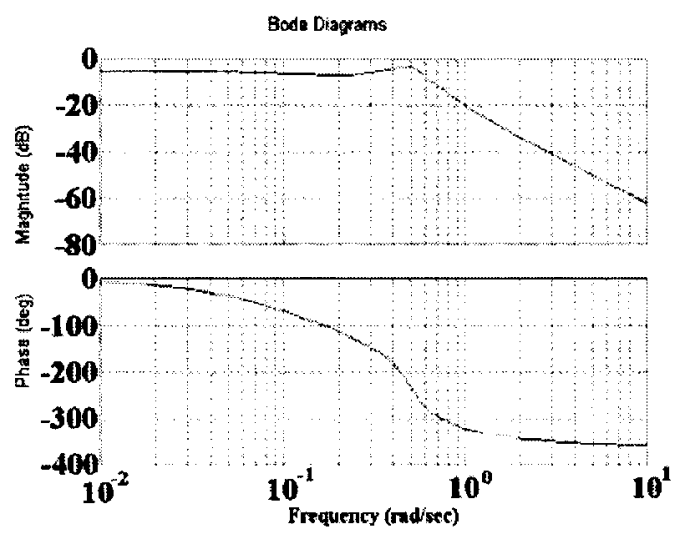

Fig. 2. Bode plot of rudder-roll transfer function. 


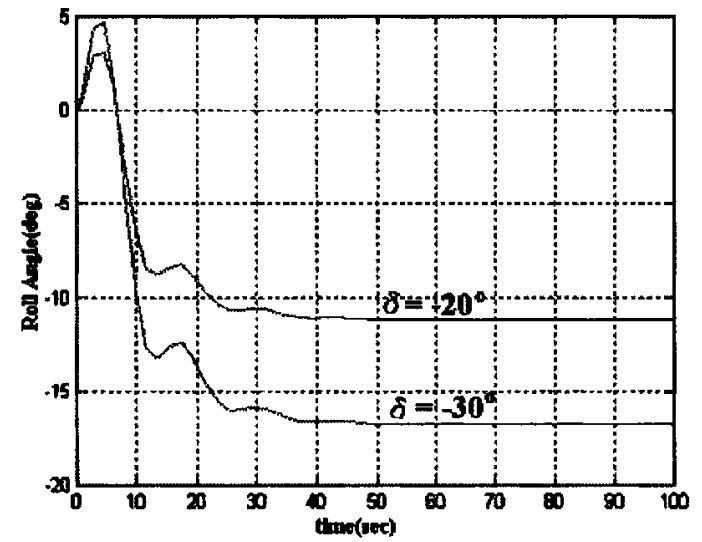

Fig. 3. Starboard turning maneuver rudder induced roll with $\delta=-20^{\circ}$ and $-30^{\circ}$.

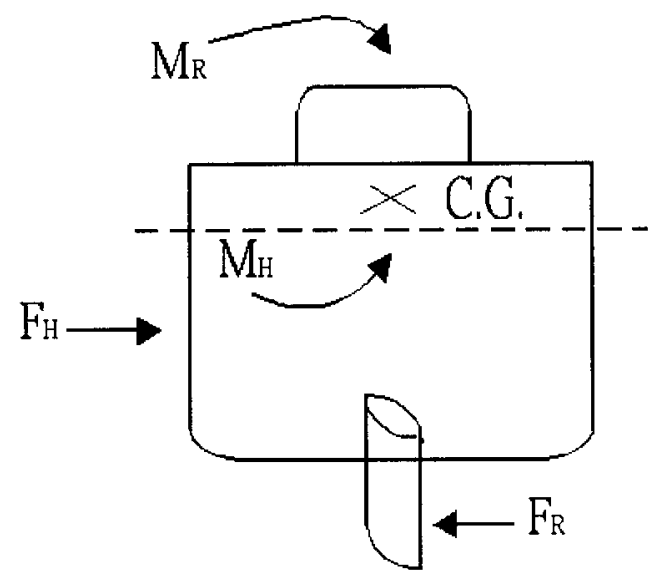

Fig. 4. Rudder induced rolling force diagram.

gradually rolls outwards, a restoring moment is generated to counteract the hull force induced moment $M_{H}$. Eventually, the ship will reach a steady outward roll angle when the restoring moment is equal but in opposite direction to the hull force induced moment $M_{H}$. The steady outward roll angle is approximately proportional to the square of the ship speed and proportional to the rudder angle but inversely proportional to the metacentric height $\overline{G M}[1]$.

It is the inward roll phenomenon caused by the rudder moment $M_{R}$ will be applied in reducing the roll motion of a ship steering in seaways. However, power of the steering gear has to be significantly increased to achieve a rudder rate up to $12 \mathrm{deg} / \mathrm{sec}$ to result in an effective RRS system.

\section{Rudder saturation and slew rate limitation}

The steering gear has associated with two non-

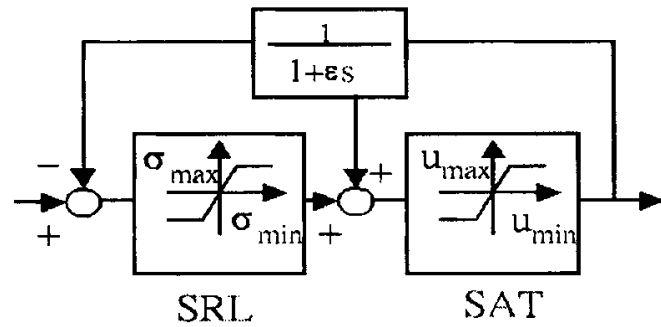

Fig. 5. Rudder saturation (SAT) and slew rate limitation (SRL) implementation.

linearities; that is, the saturation (SAT) and slew rate limitation (SRL). The rudder angles are assumed to be within \pm 30 deg and the rudder rate limits are within \pm 15 $\mathrm{deg} / \mathrm{sec}$. The rudder SAT and SRL are implemented in the structure given in Fig. 5.

The value of $\varepsilon$ in the lag box can be adjusted to represent the achievable SRL.

\section{Wave disturbance}

The wave disturbance will be modeled as an output disturbance, which is generated by passing a white noise through a second order shaping filter given by

$$
H(s)=\frac{K_{w} s}{s^{2}+2 \zeta \omega_{e} s+\omega_{e}^{2}}
$$

where $K_{w}$ is a coefficient that can be adjusted to represent wave strength effect; $\zeta$ is a damping ratio and $\omega_{e}$ is the encounter frequency. Typically, $\zeta$ is between 0.05 to 0.1 and $\omega_{e}$ is between 0.3 to $1.3 \mathrm{rad} / \mathrm{sec}$ [3]. In the simulation study, $\zeta$ is set to $0.075, \omega_{e}$ is 0.4 and $K_{w}$ is 10 , which result in a narrow band type of disturbance. It can be observed that the most often adopted PiersonMoskowitz wave spectrum does exhibit concentration of wave energy at certain frequency and the JONSWAP spectrum is even more peaked [3]. Hence, the narrow band wave disturbance is a reasonable assumption. To a first order approximation, wave motions are linear and the hull response can be obtained as a superposition of the wave induced motion and that created by rudder activity. In order to examine the effectiveness of the proposed controller, a pure sinusoidal type of wave disturbance will also be employed in the simulation study.

\section{E. Rudder roll stabilization (RRS) system structure}

The basic structure of the RRS system can be represented by the block diagram shown in Fig. 6 .

The reference signal $\phi_{\text {ref }}$ is set to zero, which indicates the desired stabilized horizontal position. The 


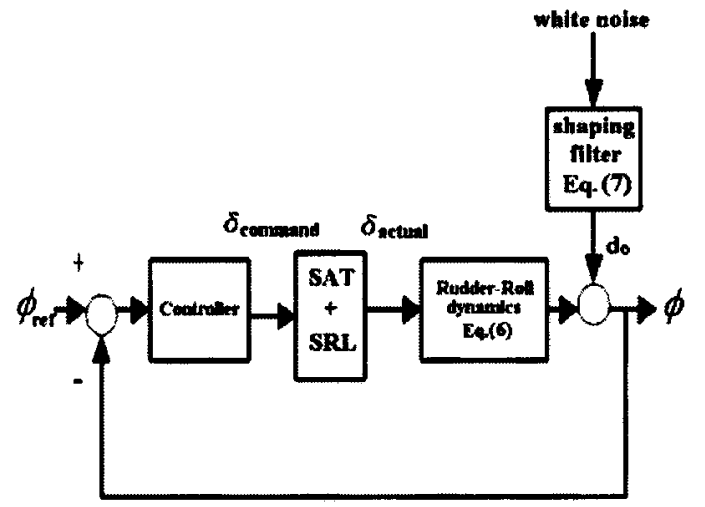

Fig. 6. RRS system, with SAT/SRL limitations.

controller $C$ will be designed with the internal model control (IMC) approach presented in latter section, and the rudder SAT and SRL will be directly taken into the design consideration.

\section{INTERNAL MODEL CONTROL (IMC) METHOD}

The IMC design method will be discussed in this section. Specifically, the advantages of IMC method will be highlighted, and the design procedure described.

\section{A. IMC parameterization}

A block diagram of the IMC structure and classical feedback structure are shown in Fig. 7A and Fig. 7B respectively. The IMC and classical feedback structures are equivalent under the following transformations:

$$
\begin{aligned}
& C=\frac{Q}{1-Q \widehat{G}} \\
& Q=\frac{C}{1+C \widehat{G}}
\end{aligned}
$$

Where $C$ is the controller, $G$ is the process being controlled, $\widehat{G}$ is the process model, and $Q$ is a design transfer function. In Figs. 7A-7B, $r$ is the reference input, $y$ is the output, $u$ is the control, $d_{i}$ is the input disturbance and $d_{o}$ is the external disturbance. Equivalence between Fig. 7A and Fig. 7B can be readily seen by transforming the dash box to a signal block and is represented by the relation shown in Eq. (8a).

The IMC structure shown in Fig. 7A shows that if there are no modeling uncertainties and there is no disturbance, there is no need of feedback. Moreover, the feedback signal in the IMC structure represents uncertainty about the process and the disturbance. Eq. (8b) implies that the controller $C$ is parameterized in terms of the design transfer function $Q$. It also indicates that the controller is directly dependent on the model $\hat{G}$.

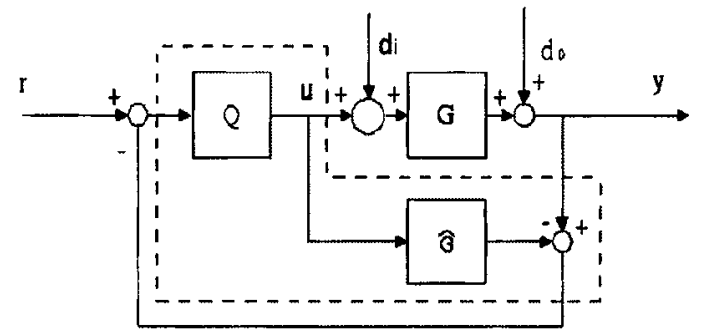

(a)

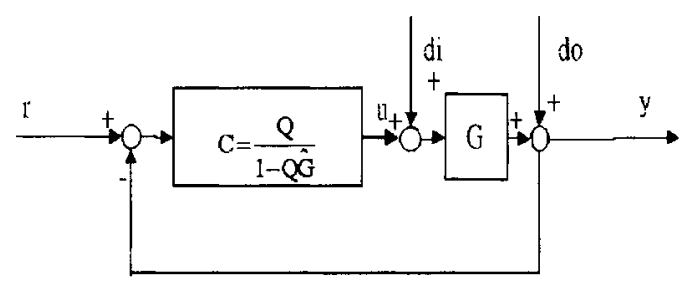

(b)

Fig. 7. (a)IMC structure, (b)Classical feedback structure.

Hence, the IMC structure can be interpreted as parameterization of the entire stabilizing controller structure, where the design transfer function $Q$ appears in Eq. (8a) plays the role of the parameter.

\section{B. IMC design procedure}

In summary, the IMC parameterization design method leads to the following controller:

$$
C=\frac{Q}{1-Q \widehat{G}}
$$

where $\widehat{G}$ is the process model and $Q$ is a design transfer function defined as

$$
Q=F \widehat{G}_{i n v}
$$

where $G_{i n v}$ is an approximation to the inverse of the process model $\widehat{G}$, and $F$ is a closed-loop specification filter, called, alternatively, the modulating filter, given in the form

$$
F=\frac{1}{(1+\beta s)^{n}}
$$

where $n$ is an integer chosen to make $Q$ bi-proper. That is, the order of the numerator is equal to the order of the denominator, and $\beta$ is a design parameter that characterizes the speed of response of the closed-loop system.

The presence of the term $G_{i n v}$ in Eq. (9b) derives from the observation that a controller with the inverse structure of the process being controlled performs perfectly under the ideal case. Specifically, $G_{i n v}$ is derived 
as follows. Assume

$$
\widehat{G}=\frac{B_{s} \cdot B_{u}}{A_{s} \cdot A_{u}}
$$

then

$$
\widehat{G}_{i n v}=\frac{A_{s} \cdot A_{u}}{\left.B_{s} \cdot\left(B_{u}\right)\right|_{s=0}}
$$

where $A_{s}, A_{u}$ represent the stable and unstable poles respectively, and $B_{s}, B_{u}$ represent the minimum phase and nonminimum phase zeros respectively. For a nonminimum phase system, direct inversion of the term $B_{u}$ is not allowed; otherwise the stability criterion will be violated. However, it is desirable to extract useful information from the term $B_{u}$ without violating the stability criterion; specially, the DC gain value of $B_{u}$ is the piece of information being retained.

\section{Internal stability}

Referring to the classical feedback structure shown in Fig. 7B, assume that $G=\widehat{G}$; that is, the process model exactly describes the process being controlled. Then the corresponding loop is called the nominal loop. It is well known that to ensure internal stability for the nominal loop, the following nominal sensitivity functions have to be stable [15]:

$$
\begin{aligned}
& T=\frac{Y}{R}=\frac{C \widehat{G}}{1+C \widehat{G}} \\
& S_{o}=\frac{Y}{D_{o}}=\frac{1}{1+C \widehat{G}} \\
& S_{i}=\frac{Y}{D_{i}}=\frac{\widehat{G}}{1+C \widehat{G}} \\
& S_{u}=\frac{U}{R}=\frac{C}{1+C \widehat{G}}
\end{aligned}
$$

where $T$ is the nominal complementary sensitivity function, $S_{o}$ is the nominal output sensitivity function, $S_{i}$ is the nominal input sensitivity function and $S_{u}$ is the nominal control sensitivity function. Satisfaction of the stability criterion by the sensitivity functions defined by Eqs. (11) ensures that between any two points in the loop, the input-output relation is stable.

Referring to the IMC structure depicted in Fig. 7A, the sensitivity functions defined by Eqs. (11) can be rewritten in terms of the design transfer function $Q$ and the model $\hat{G}$ as follows:

$$
\begin{aligned}
& T=Q \widehat{G} \\
& S_{o}=1-Q \widehat{G} \\
& S_{i}=(1-Q \widehat{G}) \widehat{G}
\end{aligned}
$$

$$
S_{u}=Q
$$

Comparing Eqs.(11) with Eqs. (12), it is seen that the sensitivity functions are nonlinear in the controller $C$ but are linear in the design transfer function $Q$. The above observation clearly indicates the advantage of the IMC structure over the classical feedback structure in terms of satisfaction of the internal stability criterion. To see this, under the IMC structure, given a stable process model $\widehat{G}$, internal stability is satisfied by choosing a stable and proper design transfer function $Q$. However, under the classical feedback structure, given a stable process model $\widehat{G}$, internal stability is not guaranteed by choosing a stable and proper controller $C$.

\section{SENSITIVITY FUNCTION-BASED APPROACH}

Referring to Fig. 7A, it follows that

$$
Y=T \cdot R+S_{0} \cdot D_{0}+S_{i} \cdot D_{i}
$$

where $Y$ is the system output, $R$ is the reference input, $D_{i}$ and $D_{o}$ are the input and output disturbances. The sensitivity function $T, S_{o}, S_{i}$ are given by Eqs. (12a)(12c).

Since the purpose of the RRS system is to maintain an upright position, the reference input is zero. Moreover, the wave disturbance will be treated as output disturbance. Hence, reduction of the wave-induced rolling motion can be achieved by proper selection of the output sensitivity function $S_{o}$ defined by Eq. (12b). Specifically, a notch filter type of sensitivity function will be selected with the center frequency located near the dominant wave frequency. In the followings, the controller design will be based on the process model $\hat{G}$ defined by the rudder-roll transfer function given by Eq. (6).

Select the output sensitivity function $S_{o}$ to have the form of a standard notch filter as

$$
S_{o}(s)=\frac{s^{2}+2 \varsigma^{*} \omega_{n} s+\omega_{n}^{2}}{\left(s+\omega_{n}\right)^{2}}
$$

It follows from Eqs.(9b), (10b) and (12b) that the modulating filter $F$ is then given by

$$
F(s)=\frac{2\left(1-\varsigma^{*}\right) \omega_{n} s}{\left.\hat{B}_{u}(s)\right|_{s=0}\left(s+\omega_{n}\right)^{2}}
$$

With $F(s)$ given by (15), the corresponding design transfer function $Q$ defined by Eq. (9b) becomes improper. However, in the IMC approach, the design transfer function $Q$ is required to be bi-proper. Hence, $F(s)$ can be modified as follows 


$$
F(s)=\frac{2\left(1-\varsigma^{*}\right) \omega_{n} s}{\left.\hat{B}_{u}(s)\right|_{s=0}\left(s+\omega_{n}\right)^{2}(s+r)^{2}}
$$

where $r$ is another design parameter to produce a biproper and stable design transfer function $Q$ given by

$$
Q=\frac{2\left(1-\varsigma^{*}\right) \omega_{n} s(1+8.2 s)\left(s^{2}+0.25 s+0.25\right)}{\left(s+\omega_{n}\right)^{2}(s+r)^{2}}
$$

The term $\left.B_{u}(s)\right|_{s=0}$ represents the DC gain of the nonminimum zero, and for our ship model defined by Eq. (6), it reduces to identity.

The corresponding output sensitivity function $S_{o}$ is derived as

$$
S_{o}(s)=\frac{N(s)}{D(s)}
$$

where

$$
\begin{aligned}
N(s) & =s^{4}+2\left(\omega_{n}+r\right) s^{3} \\
& +\left(1.26 \omega_{n}-1.26 \varsigma^{*} \omega_{n}+\omega_{n}^{2}+4 r \omega_{n}+r^{2}\right) s^{2} \\
& +\omega_{n}\left(2 r \omega_{n}+2 r^{2}+0.28 \varsigma^{*}-0.28\right) s+\omega_{n}^{2} r^{2} \\
D(s) & =\left(s+\omega_{n}\right)^{2}(s+r)^{2}
\end{aligned}
$$

In Eqs. (18), the parameters $\omega_{n}, \varsigma^{*}$, and $r$ can be treated as the design parameters that determine the shape of the notch filter.

Finally, with respect to the system transfer function given by (6) the controller can be computed from Eq. (9a) as

$$
C(s)=\frac{N_{1}(s)}{D_{1}(s)}
$$

where

$$
\begin{aligned}
N_{1}(s) & =2\left(1-\varsigma^{*}\right) \omega_{n}\left(8.2 s^{2}+s\right)\left(s^{2}+0.25 s+0.25\right) \\
D_{1}(s) & =s^{4}+2\left(\omega_{n}+r\right) s^{3} \\
& +\left(1.26 \omega_{n}-1.26 \varsigma^{*} \omega_{n}+\omega_{n}^{2}+4 r \omega_{n}+r^{2}\right) s^{2} \\
& +\omega_{n}\left(2 r \omega_{n}+2 r^{2}+0.28 \varsigma^{*}-0.28\right) s+\omega_{n}^{2} r^{2}
\end{aligned}
$$

It is to be noted that the controller given by Eqs. (19) is bi-proper. Namely, the order of the numerator is equal to that of the denominator.

\section{REFERENCE CONDITIONING TECHNIQUE}

It is well-known that saturation (SAT) and slew rate limitation (SRL) nonlinearities of control input to an open-loop stable system may cause performance degradation. Indeed, the system will grow very fast during the saturation and slew rate limitation period and may reach values that are uncontrollable with limited actuator authority [17].

It is certainly possible to design a controller that never reaches the SAT and SRL bounds; however, this will inevitably degrade the performance. In an RRS problem, it is desirable to move the rudder as quickly as possible to counteract the wave disturbance. Thus, it is necessary to bring the actuator to its SAT and SRL boundaries in order to achieve the goal.

The reference conditioning technique has been proposed to deal with saturating input for unstable linear system [18]. In this paper both the actuator SAT and SRL are considered [14]. Specifically, the reference input is modified in such a way that the controller states are consistent with actual control inputs into the plant. The control signal never attempts to take on values beyond the bound imposed by the SAT and SRL, and the linear design properties are preserved.

\section{A. Rudder saturation conditioning}

The configuration of the reference conditioning filter is presented as follows. Given a bi-proper controller $C$ described by Eqs. (19), it is possible to describe the controller in terms of a strictly proper term and a feedthrough term as follows:

$$
C=C_{0}+C_{1}
$$

where $C_{0}$ is the feedthrough term and $C_{1}$ is the strictly proper term. Let $\phi$ be the actual roll angle and let $\mathrm{R}$ be the reference angle. Consider the following control law:

$$
\begin{aligned}
u^{*} & =C(R-\phi) \\
& =C_{0}(R-\phi)+C_{1}(R-\phi)
\end{aligned}
$$

The control input based on Eq. (21) may lead to saturation and slew rate limitation if the actual roll angle $\phi$ is very different from the reference angle $R$. Thus, it is possible to choose a new reference angle to avoid the above condition.

Let us assume for this moment there is only SAT limitation. Let $R_{n e w}$ be the modified reference angle, which can be calculated by solving for the following relation:

$$
\begin{aligned}
& C_{1}\left(R_{\text {new }}-\phi\right)+C_{0}\left(R_{\text {new }}-\phi\right) \\
& \quad=\operatorname{SAT}\left[C_{1}\left(R_{\text {new }}-\phi\right)+C_{0}(R-\phi)\right]
\end{aligned}
$$


where $S A T[\bullet]$ is a saturation function. Solving Eq. (22) for $R_{\text {new }}$, we have

$$
\begin{aligned}
R_{\text {new }} & =\frac{1}{C_{0}}\left\{\operatorname{SAT}\left[C_{1}\left(R_{\text {new }}-\phi\right)-C_{0} \phi+C_{0} R\right]\right. \\
& \left.-\left[C_{1}\left(R_{\text {new }}-\phi\right)-C_{0} \phi\right]\right\}
\end{aligned}
$$

Rearranging Eq. (23), it follows that

$$
R_{\text {new }}=\frac{1}{C_{0}}\left\{S A T\left[u_{1}+C_{0} R\right]-u_{1}\right\}
$$

where

$$
u_{1}=C_{1}\left(R_{\text {new }}-\phi\right)-C_{0} \phi
$$

Note that the left-hand side (LHS) of Eq. (22) is the actual input into the plant that will not activate the saturation nonlinearity.

Let us call the LHS of Eq. (22) $u$ and write

$$
u=C_{1}\left(R_{\text {new }}-\phi\right)+C_{0}\left(R_{\text {new }}-\phi\right)
$$

Substitution of Eq. (25) into Eq. (26) gives

$$
u=u_{1}+C_{0} R_{\text {new }}
$$

The relationships described by Eqs. (22)-(27) can be realized in terms of the configuration shown in Fig. 8.

\section{B. Rudder saturation and slew rate limitation condition- ing}

If both saturation and slew rate limitation are considered, Eq. (24) is modified as

$$
R_{\text {new }}=\frac{1}{C_{0}}\left\{(S A T+S R L)\left[u_{1}+C_{0} R\right]-u_{1}\right\}
$$

where $(S A T+S R L)\left[u_{1}+C_{0} R\right]$ implies that both the saturation and slew rate limitation constraints are imposed on the term $u_{1}+C_{0} R$. The conditioning scheme

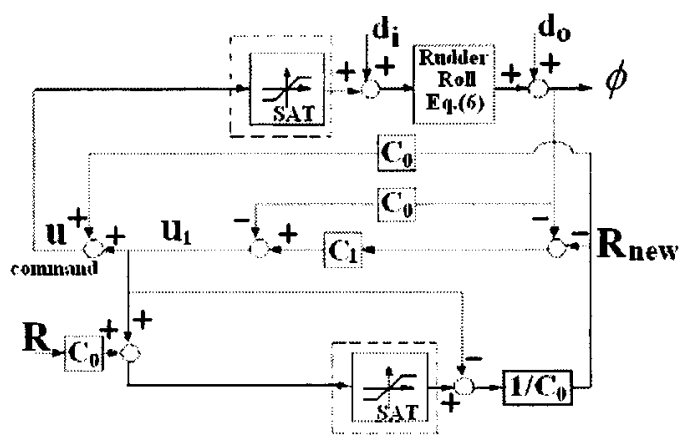

Fig. 8. Controller realization with SAT. with $S A T$ and $S R L$ constraints is realized with the configuration shown in Fig. 9.

To take into account the presence of $S A T$ and $S R L$ in the system, one would like to 'alert' the controller whenever either one of these non-linearities is activated. At this point the controller should stop 'pushing', since either the rudder reaches its motion limit or its motion rate limit. This is accomplished by passing the control signal through the system presented in the dash line rectangle shown in Fig. 9.

Summarizing, the control state is now calculated based on the difference between $R_{\text {new }}$ and $\phi$. This ensures the control state is always consistent with the actual input into the plant. Thus, it is possible to execute the RRS very quickly by complying with limited actuator power, without introducing the saturation and slew rate limitation nonlinearities.

\section{NUMERICAL SIMULATIONS}

Recall that for the wave model generated by passing white noise sequence through a shaping filter defined by Eq. (7). In the following computations, the damping ratio $\zeta$ is set to 0.075 , the encounter frequency $\omega_{e}$ is set to $0.4 \mathrm{rad} / \mathrm{sec}$ and the wave strength factor $K_{w}$ is set to 10 , which will give a wave-induced roll motion with root mean square (RMS) value around $12 \mathrm{deg}$. The wave shaping filter employed in the simulation study is then given by

$$
H(s)=\frac{10 s}{s^{2}+0.06 s+0.16}
$$

The design parameters that determine the characteristics of the output sensitivity functions defined by Eq. (18) are selected as follows. Specifically, $\omega_{n}=0.08$,

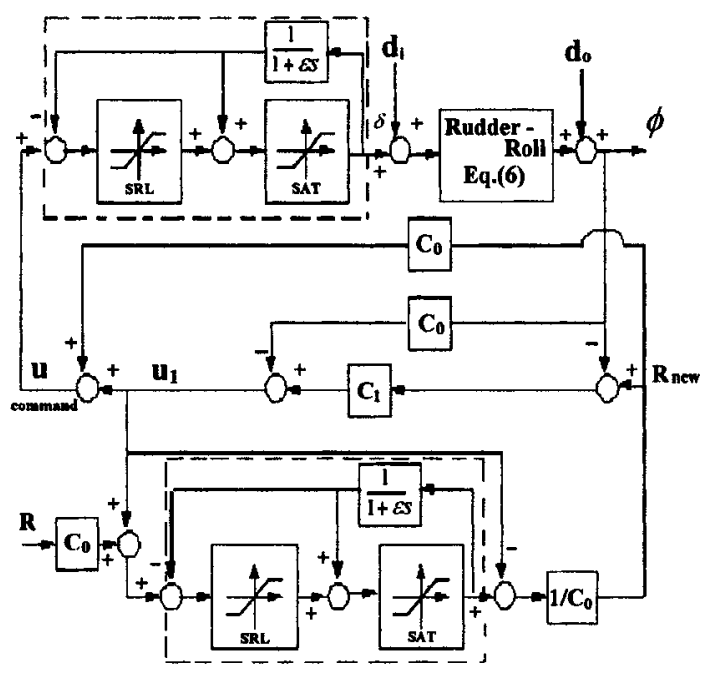

Fig. 9. Controller realization with SAT and SRL. 
$\varsigma^{*}=11.9$ and $r=1$.

The corresponding output sensitivity function $S_{0}$ of our design is then given by

$$
S_{o}(s)=\frac{s^{4}+2.16 s^{3}+0.2277 s^{2}+0.417 s+0.0064}{s^{4}+2.16 s^{3}+1.3264 s^{2}+0.1728 s+0.0064}
$$

Finally, the controller $C(s)$ defined by Eq. (19) can be computed as

$$
C(s)=\frac{-14.3008 s^{4}-5.3192 s^{3}-4.0112 s^{2}-0.436 s}{s^{4}+2.16 s^{3}+0.2277 s^{2}+0.417 s+0.0064}
$$

The Bode plot of the output sensitivity function $S_{0}(s)$ defined by Eq. (30) is shown in Fig. 10. It can be seen that the center frequency of the notch is located near $0.4 \mathrm{rad} / \mathrm{sec}$. Hence, an effective roll reduction can be expected for wave disturbance with dominating frequency around $0.4 \mathrm{rad} / \mathrm{sec}$.

It is to be noted that as the magnitude of the output sensitivity function $S_{o}$ is pushed down near the dominating encounter wave frequency, it pops up at some other frequency regions. This is the so-called water bed effect, which characterizes the nonminimum phase systems. Consequently, inevitable trade-off exists in the RRS controller design. Namely, attenuating the wave disturbance near the center frequency is accompanied by magnifying of the wave disturbance at other frequency. Hence, careful design of the notch filter to capture the dominating frequency is very important to achieve good roll stabilization performance.

To illustrate the feasibility of the proposed RRS design method, a pure sinusoidal type of disturbance with an amplitude of $15^{\circ}$, frequency of $0.4 \mathrm{rad} / \mathrm{sec}$ is taken as the output disturbance. The stabilized results are given in Fig. 11A and the rudder angle employed is given in Fig. 11B. It is clear that for the simple sine wave disturbance, very good roll reduction is achieved with minimal control effort required. Specifically, the

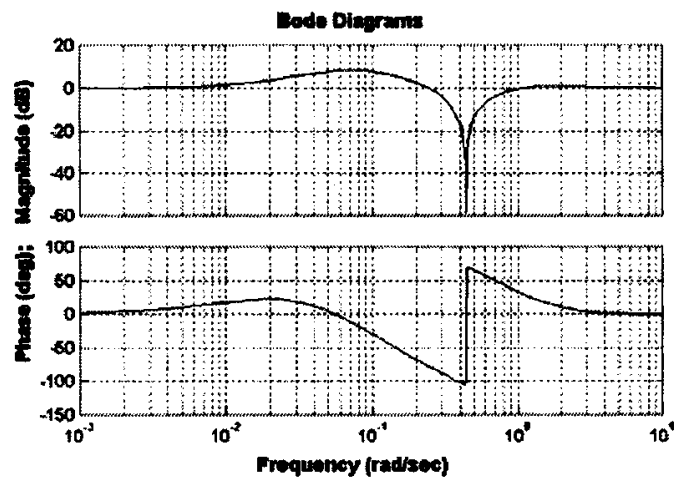

Fig. 10. Bode plot of the output sensitivity function So(s). roll motion amplitude is reduced from $15^{\circ}$ to $3^{\circ}$ and the rudder angle command is within $\pm 30^{\circ}$.

The more realistic narrow band type of wave disturbance generated by passing a white noise sequence through the shaping filter defined by Eq. (29) is also examined to evaluate the performance of the proposed RRS system. To give a benchmark performance of the proposed controller, it is first assumed that there is no SAT or SRL limitation on the rudder. Fig. 12A shows the roll angle history with and without the controller and Fig. 12B gives the rudder angle time history. It is seen that the roll angle has been reduced significantly. Specifically, the root mean square (RMS) value of the roll angle has been reduced from $12.5^{\circ}$ to $4.5^{\circ}$, which corresponds to a $66 \%$ roll angle reduction ratio defined as follows:

Roll reduction ratio

$$
=\frac{R M S_{\text {controller off }}-R M S_{\text {controller on }}}{R M S_{\text {controller off }}}
$$

However, the rudder angle demand is well beyond the SAT and SRL boundaries. The RMS value of the rudder angle is $28.9 \mathrm{deg}$.

Figs. 13-14 give the simulation results by taking

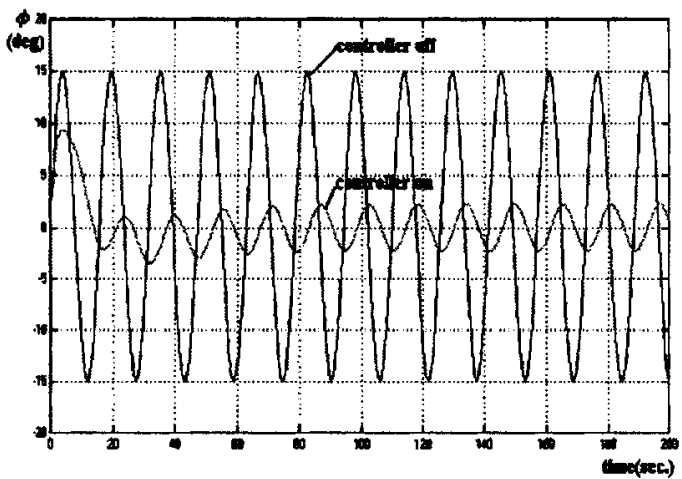

(a)

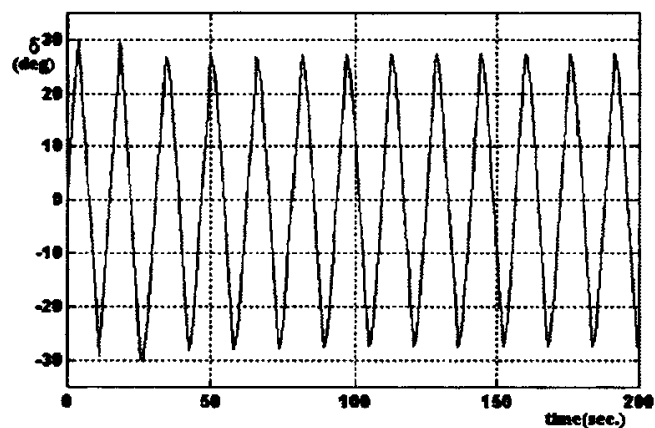

(b)

Fig. 11. (a)Roll reduction for sinusoidal disturbance; (b)Rudder angle for sinusoidal disturbance. 


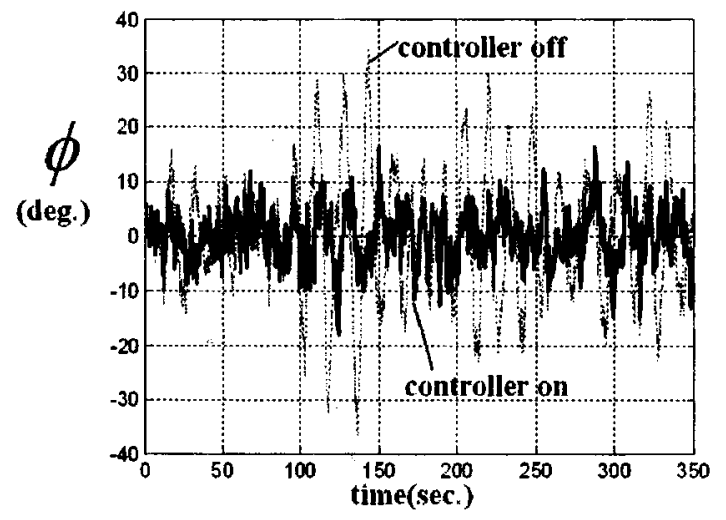

(a)

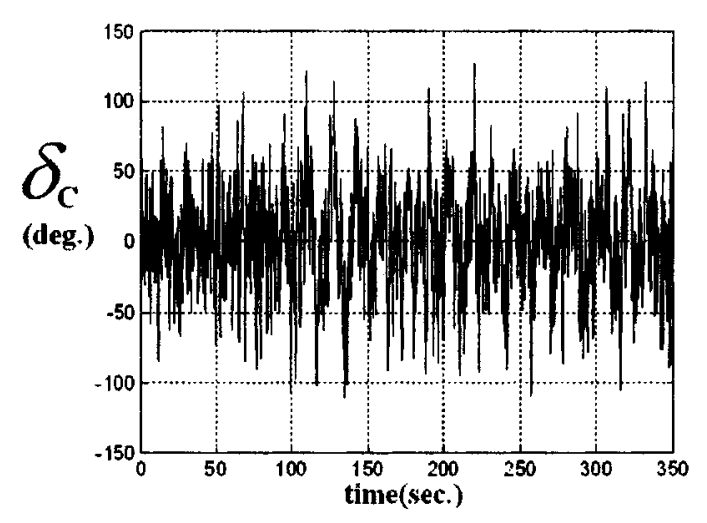

(b)

Fig. 12. (a)Roll reduction for narrow band disturbance, no SAT/SRL constraints; (b)Rudder angle for narrow band disturbance, no SAT/ SRL constraints.

the limited actuator authority into consideration based on the block diagram of Fig. 6. Specifically, the rudder command generated from the controller is compared with the SAT and SRL constraints. If the rudder command is within the SAT and SRL boundaries, the rudder command is directly taken as the actual rudder input. However, if the rudder command goes beyond the SAT or SRL bound, the actual rudder input will be set to the SAT or SRL boundary value. It is to be noted that in this case, the SAT and SRL are not taken directly into the controller design consideration. Namely, the controller is not aware of the limited actuator authority. Hence, a large rudder command beyond the SAT and SRL might be generated.

Specifically, Fig. 13A shows the roll angle history with rudder $S A T= \pm 30^{\circ}$ and $S R L= \pm 12^{\circ} / \mathrm{sec}$, and Fig. $14 \mathrm{~A}$ shows the roll angle history with $S R L= \pm 30^{\circ}$ and $S R L= \pm 15^{\circ} / \mathrm{sec}$. The roll angle reduction ratio achieved is $28.5 \%$ for $S R L= \pm 12 \% \mathrm{sec}$ and is $32.2 \%$ for $S R L=$ $\pm 15 \% \mathrm{sec}$. This is in agreement with the general observation that the RRS performance improves as the rudder

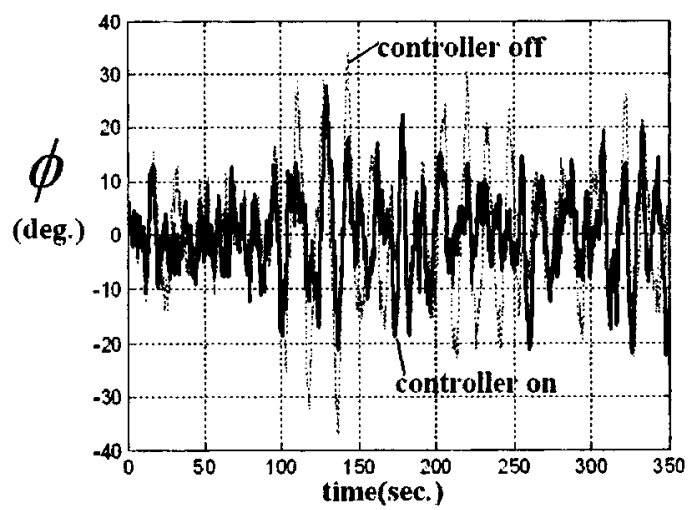

(a)



(b)

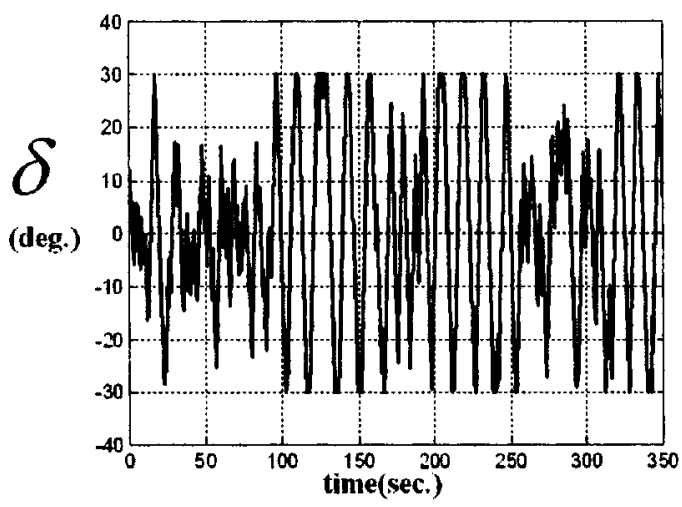

(c)

Fig. 13. (a)Roll reduction for narrow band disturbance, $S R L= \pm 12 \% \mathrm{sec}$, without reference conditioning; (b)Rudder command for narrow band disturbance, $S R L= \pm 12 \% \mathrm{sec}$, without reference conditioning; (c)Actual rudder angle for narrow band disturbance, $S R L= \pm 12^{\circ}$ /sec, without reference conditioning.

rate increases.

As can be seen from Fig. 13B and Fig. 14B that the rudder command is well beyond the $S A T$ and $S R L$ boundaries. Hence, the actual rudder angles are reduced to comply with the steering gear $S A T$ and $S R L$ constraints.

Figs. 15-16 show the stabilized results of the 


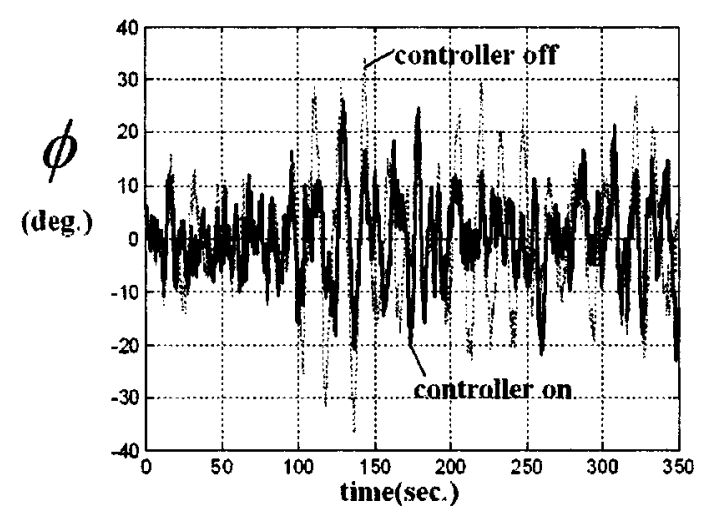

(a)

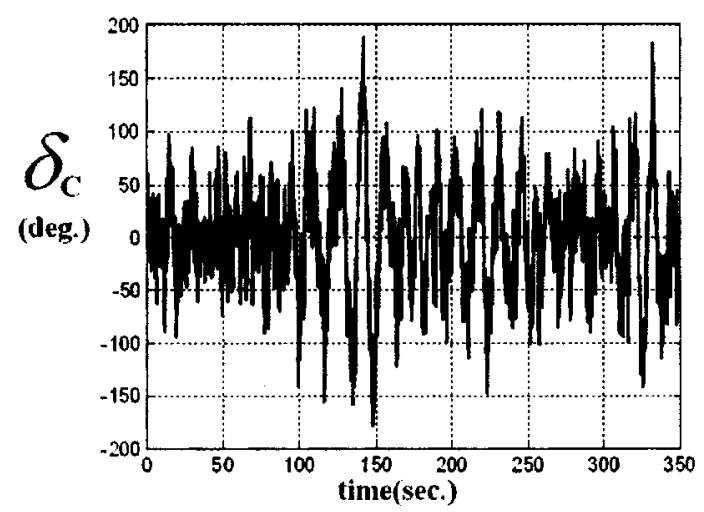

(b)

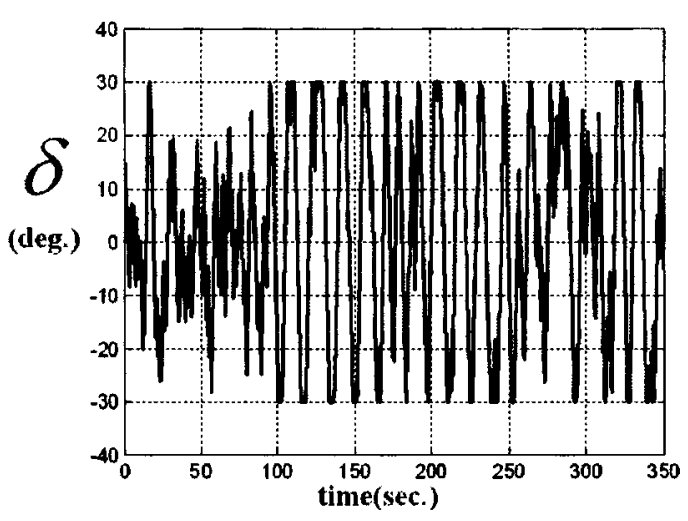

(c)

Fig. 14. (a)Roll reduction for narrow band disturbance, $S R L= \pm 15 \% \mathrm{sec}$, without reference conditioning; (b)Rudder command for narrow band disturbance, $S R L= \pm 15^{\circ} / \mathrm{sec}$, without reference conditioning; (c)Actual rudder angle for narrow band disturbance, $S R L= \pm 15^{\circ}$ /sec without reference conditioning.

proposed controller design method by using the reference conditioning technique in taking the actuator SAT and SRL into the design consideration. Same as previous simulations, the maximum rudder angle is limited within $\pm 30^{\circ}$ and the maximum rudder rate is $\pm 12 \% \mathrm{sec}$

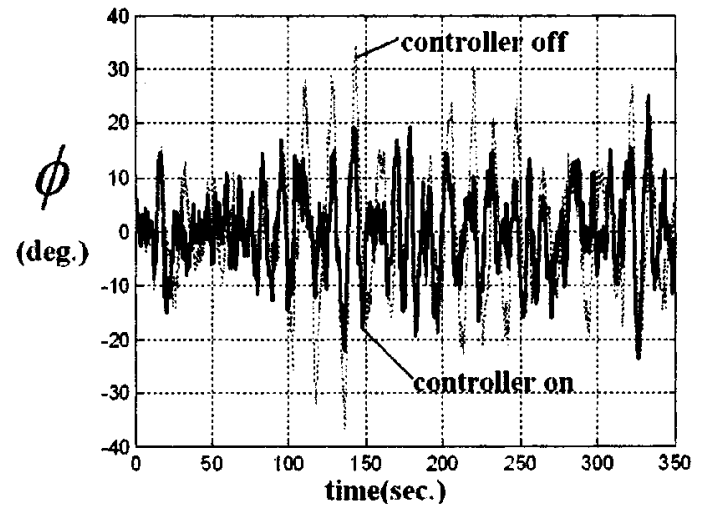

(a)

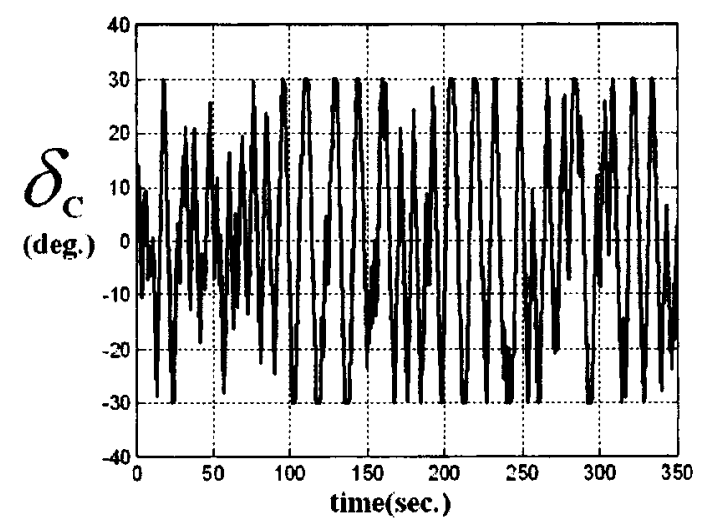

(b)

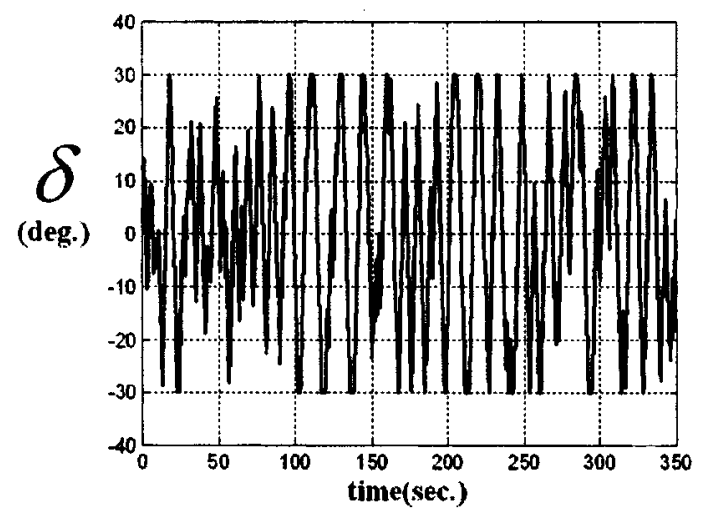

(c)

Fig. 15. (a)Roll reduction for narrow band disturbance, $S R L= \pm 12 \% \mathrm{sec}$, with reference conditioning; (b)Rudder command for narrow band disturbance, $S R L= \pm 12 \%$ sec, with reference conditioning; (c) Actual rudder angle for narrow band disturbance, $S R L= \pm 12 \%$ sec, with reference conditioning.

and $\pm 15 \%$ sec respectively. Specifically, Figs. 15 correspond to $\pm 12 \%$ sec SRL and Figs. 16 correspond to $\pm 15 \%$ sec SRL. The roll reduction ratio is $36.4 \%$ for a SRL of $\pm 12 \% \mathrm{sec}$, and the roll reduction ratio is $49.1 \%$ for the SRL of $\pm 15 \%$ sec. 


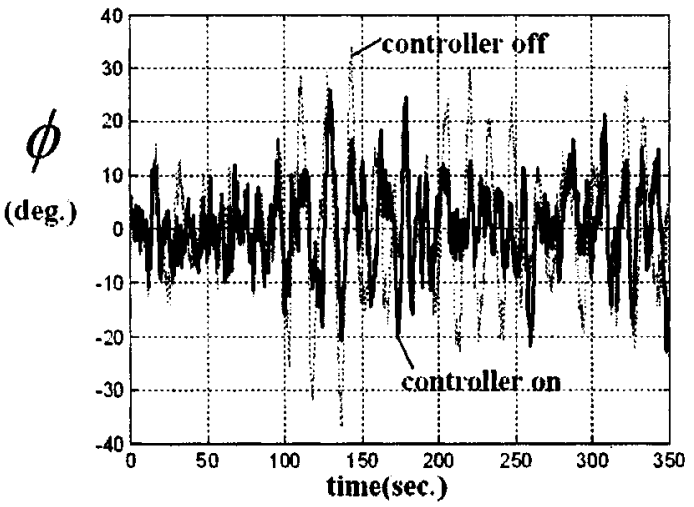

(a)

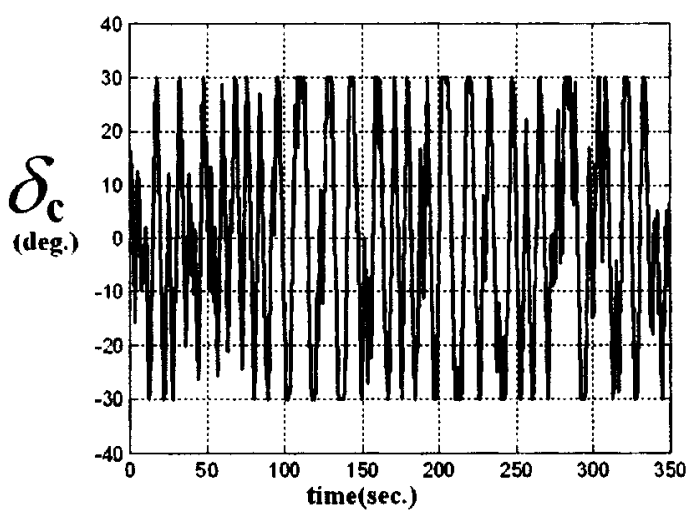

(b)

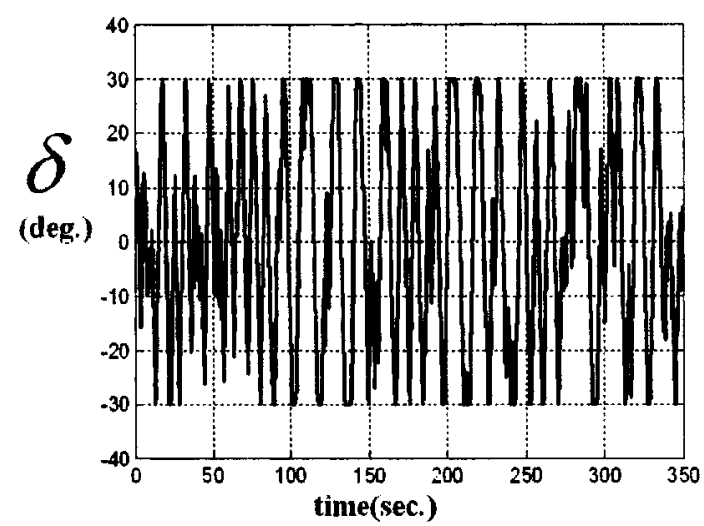

(c)

Fig. 16. (a)Roll reduction for narrow band disturbance, $S R L= \pm 15 \%$ sec, with reference conditioning; (b)Rudder command for narrow band disturbance, $S R L= \pm 15^{\circ} / \mathrm{sec}$, with reference conditioning; (c)Actual rudder angle for narrow band disturbance, $S R L= \pm 15^{\circ} / \mathrm{sec}$, with reference conditioning.

By comparing the rudder commands of Figs. 15B $-16 \mathrm{~B}$ with those of Figs.13B-14B, it can be observed that the rudder angle commands are within the SAT and SRL boundaries while employing the reference conditioning technique, which takes the limited actuator authority into the design consideration.

Moreover, the roll reduction performance has improved when using the reference conditioning technique. Specifically, for $\pm 12 \% \mathrm{sec}$ SRL, the reduction ratio is improved from $28.5 \%$ to $36.4 \%$ and for $\pm 15 \%$ sec SRL, the reduction ratio is improved from $32.2 \%$ to $49.1 \%$.

Table 1 summarizes the roll reduction results discussed in the numerical examples using different SRL values and with or without the reference conditioning techniques. The roll reduction ratio is larger when a higher SRL is employed. Besides, the advantage of using the reference conditioning technique is clearly seen from the table.

\section{CONCLUSIONS}

An IMC-based rudder roll stabilization controller design method is found appealing in terms of wave disturbance rejection. Specifically, under the IMC framework, the sensitivity functions depend linearly on the design transfer function $Q$. This allows us to directly shape the output sensitivity function. The design parameter of the proposed approach has much more clear physical meaning than the popular LQ optimal control, where the weighting matrices of the cost function being minimized play the role of the design parameters. It is also more convenient than the $H \infty$ control approach that shapes the weighting matrices to form a bound on the sensitivity functions. The adopted reference conditioning technique allows the controller to exert its full power without violating the SAT and SRL boundaries imposed by limited actuator authority.

Due to the ever changing sea state conditions, further research on on-line estimation of the dominating encounter wave frequency is required and the center frequency of the notch filter should be adjusted accordingly to make the proposed controller adaptive to different sea states. If a confused sea state is encountered; namely, no dominant frequency exists at all, then the RRS would be of little use and should be turned off. This is because a very wide notch in the output sensitivity function is needed and the depth of the notch would be very shallow. Consequently, the attenuation effect would be very small.

\section{REFERENCES}

1. E.V. Lewis, Editor, "Principles of Naval Architecture, Vol. III, Motion in Waves and Controllability", The Society of Naval Architects and Marine Engineers, Jersey City, New Jersey, 1989.

2. W.E. Cowley and T.H. Lambert, "Sea trials on a roll stabilizer using the ship's rudder", Proceedings, $4^{\text {th }}$ Ship 
Table 1. Roll reduction results summary

\begin{tabular}{lccc}
\hline & Controller off (roll angle) & Controller on (roll angle) & Roll reduction ratio \\
\hline No saturation & $12.5 \mathrm{deg}$ & $4.5 \mathrm{deg}$ & $66 \%$ \\
No reference conditioning $S R L= \pm 12 \% \mathrm{sec}$ & $12.5 \mathrm{deg}$ & $8.94 \mathrm{deg}$ & $28.5 \%$ \\
No reference conditioning $S R L= \pm 15 \% \mathrm{sec}$ & $12.5 \mathrm{deg}$ & $8.48 \mathrm{deg}$ & $32.2 \%$ \\
Reference conditioning $S R L= \pm 12 \% \mathrm{sec}$ & $12.5 \mathrm{deg}$ & $7.95 \mathrm{deg}$ & $36.4 \%$ \\
Reference conditioning $S R L= \pm 15 \% \mathrm{sec}$ & $12.5 \mathrm{deg}$ & $6.36 \mathrm{deg}$ & $49.1 \%$ \\
\hline
\end{tabular}

Control Systems Symposium, The Hague, The Netherlands, 1975, pp. 2.195-2.213.

3. T.I. Fossen, "Guidance and Control of Ocean Vehicles", John Wiley and Sons, New York, New York,1994.

4. G.N. Roberts, "A note on the applicability of rudder roll stabilization for ships", Proceedings, American Control Conference, San Francisco, California, 1993, pp. 24032407.

5. G.N. Roberts, "A method to determine the applicability of rudder roll stabilization for ship", Proceedings, $12^{\text {th }}$ IFAC World Congress, Sydney, Australia, 1993, pp. 5. 405-5.408.

6. M.R. Katebi, D.K. Wong and M.J. Grimble, "LQG autopilot and rudder roll stabilization control system design", Proceedings, $8^{\text {th }}$ Ship Control Systems Symposium, Bath, U.K, 1987, pp. 3.69-3.84.

7. J. Stoustrup, H.H. Niemann and M. Blanke, "Roll damping by rudder control-a new $\mathrm{H} \infty$ approach", Proceedings, $3^{\text {rd }}$ IEEE Control Applications Conference, Glasgow, U. K., 1994, pp. 839-844.

8. J. van Amerongen and H.R. van Nauta Lemke, "Adaptive control aspects of a rudder roll stabilization system", Proceedings, $10^{\text {th }}$ IFAC World Congress, Munich, Germany, 1987, pp. 215-219.

9. M.T. Sharif, G.N. Roberts and R. Sutton, "Robust fin/ rudder ship roll stabilization", Proceedings, $3^{\text {rd }}$ IEEE Control Applications Conference, Glasgow, U.K., 1994, pp. 1107-1112.

10. C.G. Kallstrom, P. Wessel and S. Sjolander, "Roll reduction by rudder", Proceedings, the Society of Naval Architects and Marine Engineers, Spring Meeting, Pittsburgh, Pennsylvania, 1988, pp. 67-76.

11. E. Batis, D.A. Woolaver and T.A. Back, "Rudder roll stabilization for coast guard cutters and frigates", Naval Engineer Journal, May, 1983, pp. 267-282.

12. J. van Amerongen, P.G.M. van der Klugt and H.R. van Nauta Lemke, "Rudder roll stabilization for ships", Automatica, Vol. 26, No. 4, 1990, pp. 679-690.

13. R.G.A. Melville, C. Kallstrom and K. Theoren, "Rudder roll stabilization - an improved control law", Proceedings, $3^{\text {rd }}$ IEEE Control Applications Conference, Glasgow, U.
K., 1994, pp. 1099-1105.

14. C.Y. Tzeng, G.C. Goodwin, and S. Crisafulli, "Internal Model Autopilot Design with Saturation and Slew Rate Limitation Actuator", International Shipbuilding Progress, Vol. 46, No. 448, 1999, pp. 401-420.

15. M. Morari and E. Zafiriou, "Robust Process Control", Prentice-Hall, Englewood Cliffs, N.J., 1989.

16. W.Y. Hwang, "Cancellation effect and parameter identifiability of ship steering dynamics", International Shipbuilding Progress, Vol.26, No. 332, 1982, pp. 90-120.

17. K.J. Astrom and B. Wittenmark, "Adaptive Control", Addison-Wesley, Reading, Massachusetts, 1995.

18. M. Seron, G.C. Goodwin and S.F. Graebe, "Control System Design Issues for Unstable Linear Systems with Saturating Inputs" IEE Proceedings, Part D, Vol. 142, No. 4, 1995, pp. 335-344.

\section{依據露敏度函數特性設計横搖稳定 舵控制器}

曾慶耀吴重毅朱御龍 海洋大學航運技術研究所

摘 要

船舶航行於海中, 遭遇波浪干擾所產生之横搖 運動, 可視爲系統遭受輸出干擾之影響, 因此可針對 系統之輸出露敏度函數著手設計控制器, 以達到抑制 波浪千擾之目的。本文使用内模式控制之設計架構, 籍由設計之轉移函數反推而求得系統之露敏度函數, 將可易於滿足系統内部穞定之條件。於設計過程中, 使用參考信號調節法, 考虑舵機系統之舵角極限及舵 機速率限制於控制器設計之中, 將可避免控制器下達 超過舵機系統工作能力之控制命令。經由模擬驗證, 控制器對正弦波形式之波浪千擾及具有窄頻特性之波 浪千擾, 皆有良好之抑制效果。 